\title{
LETTER
}

\section{Inefficient Star Formation In Extremely Metal} Poor Galaxies

\author{
Yong $\mathrm{Shi}^{1,2}{ }^{1, \text { Lee Armus }^{3} \text {, George Helou }}{ }^{3}$, Sabrina Stierwalt ${ }^{4}$, Yu Gao ${ }^{5}$, Junzhi Wang ${ }^{6}$, Zhi-Yu Zhang ${ }^{7}$, Qiusheng Gu ${ }^{1,2}$
}

Thd first galaxies contain stars born out of gas with little or no metals. The lack of metals is expected to inhibit efficient gas cooling and star formation ${ }^{1.21}$ but this effect has yet to be observed in galaxies with oxygen abundance relative to hydrogen below a tenth of that of the Sun ${ }^{24}$. Extremely metal poor nearby galaxies may be our best local laboratories for studying in detail the conditions that prevailed in low metallicity galaxies at early epochs. Carbon Monoxide ( $\mathrm{CO}$ ) emission is unreliable as tracers of gas at low metallicities 5.7 , and while dust has been used to trace gas in lowmetallicity galaxies $\sqrt{5 / 8.10}$, low-spatial resolution in the far-infrared has typically led to large uncertainties 5 .10. Here we report spatiallyresolved infrared observations of two galaxies with oxygen abundances below 10 per cent solar, and show that stars form very inefficiently in seven star-forming clumps of these galaxies. The star formation efficiencies are more than ten times lower than found in normal, metal rich galaxies today, suggesting that star formation may have been very inefficient in the early Universe.

The two galaxies that are the focus of this study are Sextans A, a dwarf irregular at $1.4 \mathrm{Mpc}$ with oxygen abundance of $7 \%$ Solar ${ }^{\sqrt{11,12}}$, and ESO 146-G14, a low-surface-brightness galaxy at $22.5 \mathrm{Mpc}$ with $9 \%$ solar oxygen abundance ${ }^{11,13}$. Their metallicities may be similar to that of gas out of which Population II stars form in the early Universe around redshift from 7 to $12^{14}$. An effective way to estimate the total gas content in extremely metal poor galaxies is to employ spatially resolved maps of the far-infrared (IR) emitting dust as tracers of the atomic and molecular gas by multiplying with an appropriate gas-todust ratio (GDR) that is inferred from regions with little or no active star formation, a methodology that has been extensively demonstrated in relatively metal rich galaxies ${ }^{7 / 15}$.

The infrared observations described in this paper were carried out at 70, 160, 250, 350 and $500 \mu \mathrm{m}$ with the Photodetector Array Camera and Spectrometer (PACS $)^{16}$ and Spectral and Photometric Imaging REceiver (SPIRE) $)^{17}$ onboard the Herschel Space Observatory. We complement our far-IR data with mid-IR images from the Spitzer Space Telescope to construct the full IR spectral energy distributions (SED) Far-ultraviolet (UV) images from the GALEX Space Telescope archive are used to trace un-obscured star formation. Maps of atomic gas are available in the literature for Sextans $A^{18}$ and ESO 146-G14 ${ }^{19}$.

Figure 1 shows the multi-wavelength images of our sample galaxies. Based on the far-UV image we defined the star-forming disk as an ellipse to closely follow the 10- $\sigma\left(\sim 26 \mathrm{AB}-\mathrm{mag} / \mathrm{arcsec}^{2}\right)$ contour of the far-UV emission as shown in the Fig. 1 and listed in Table 1. Individual star-forming clumps within the disk are identified as circled re-

${ }^{1}$ School of Astronomy and Space Science, Nanjing University, Nanjing 210093, China. ${ }^{2} \mathrm{Key}$ Laboratory of Modern Astronomy and Astrophysics (Nanjing University), Ministry of Education, Nanjing 210093, China. ${ }^{3}$ Infrared Processing and Analysis Center, California Institute of Technology, 1200 E. California Blvd, Pasadena, CA 91125, USA. ${ }^{4}$ Department of Astronomy, University of Virginia, P.O. Box 400325, Charlottesville, VA 22904, USA. ${ }^{5}$ Purple Mountain Observatory, Chinese Academy of Sciences, 2 West Beijing Road, Nanjing 210008, China. ${ }^{6}$ Shanghai Astronomical Observatory, Chinese Academy of Sciences, 80 Nandan Road, Shanghai 200030, China. ${ }^{7}$ Institute fo Astronomy, University of Edinburgh, Royal Observatory, Blackford Hill, Edinburgh EH9 3HJ, UK gions with elevated $(>3 \sigma)$ emission relative to local disk backgrounds in both far-UV and $160 \mu \mathrm{m}$ bands after smoothing images to 28 arcsec resolutions. The diffuse emission is measured by subtracting the total emission of all star-forming clumps in the disk from the integrated disk emission. For Sextans A, we also identified several individual diffuse regions that show extended emission in 70 and $160 \mu \mathrm{m}$ bands but with surface brightness below $3 \sigma$ of local disk backgrounds. In order to derive the dust mass, including both diffuse and clumped components, we fit the IR SED with a standard dust model 20 . The best-fit SEDs are shown in Fig. 2, and derived dust masses are listed in Table 1.

With spatially resolved dust and HI maps, the total gas masses of individual star-forming clumps can be derived by multiplying their dust masses with the appropriate GDR based on regions with little or no star formation. As is usually done for nearby galaxies ${ }^{7 / 15}$ the GDR in the star-forming clumps is taken as the ratio of atomic gas to dust in the diffuse region of the disk. This works because (1) the atomic gas dominates the total gas mass in the diffuse regions, and (2) the GDR is roughly constant in star-forming disks after removing the metallicity gradients $15 \sqrt{21}$. Dwarf galaxies in general show little or no metallicity gradients across their disks $\frac{22}{2}$, including Sextans A, which has a variation of less than $0.1 \mathrm{dex}$. Table 1 lists the derived gas masses of individual star-forming clumps corrected for inclination based on the GDR of the integrated diffuse emission $\left(G D R=1.4 \times 10^{4}\right.$ for Sextans A and 4,400 for ESO 146-G14). For Sextans A, three individual diffuse regions have similar GDR's that are only a factor of 1.5-2 lower than the one derived from the integrated diffuse emission. Adopting the GDR of 140 at the solar metallicity ${ }^{20}$, our derived GDR of diffuse regions of two galaxies scales roughly with the metallicity as $1 / Z^{1.5-1.7}$. For each star-forming clump, the star formation rate (SFR) is estimated by combining the FUV-based (unobscured) and $24 \mu \mathrm{m}$-based (obscured) SFRs ${ }^{23}$. The uncertainties in the derived gas masses and SFRs are estimated to be around 0.3 dex and 0.2 dex, respectively.

Figure 3 shows the distribution of seven dusty star-forming clumps in the plane of SFR surface densities vs. total gas mass surface densities, compared to spirals and merging galaxies 3 . In sharp contrast to deriving the gas surface density from the HI gas alone (open symbols in the figure), when using the dust to estimate the total gas, the metal-poor star-forming clumps appear to have significantly lower star formation efficiencies (SFEs) compared to those found in metal-rich galaxies, or those derived for the clumps using the HI gas alone. Four extremely metal poor clumps in Sextans A show almost two orders of magnitude lower SFEs compared to spirals when measured over the similar physical scales. This result still holds if we adopt a GDR of individual diffuse regions, which causes the gas densities only drop by $0.2-0.3$ dex. For ESO 146-G14, one star-forming clump shows significantly (100) lower SFEs and the remaining two have SFEs about a factor of 10 lower than spirals at sub-kpc scales and similar gas densities. If any dark molecular gas is present in the diffuse region, the derived SFEs would be even lower. For our seven metal poor clumps as a group, the K-S test indicates a probability of only $10^{-4}$ that their SFRs have 

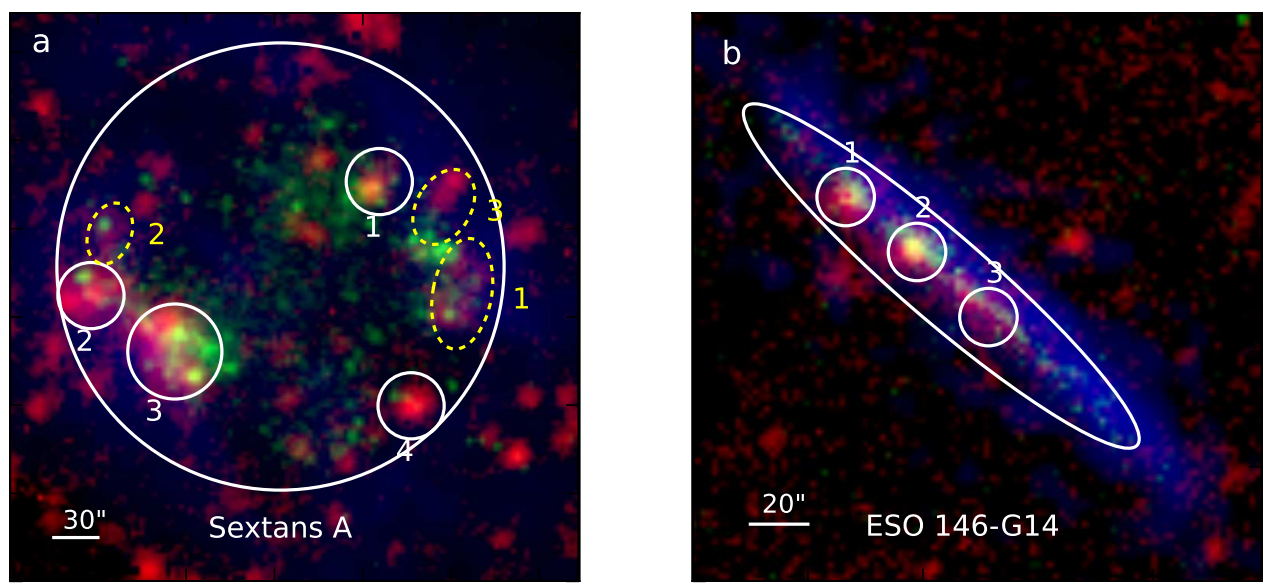

Figure 1 False-color, multi-wavelength images of our sample galaxies. a, Images of Sextans A: red, the sum of Herschel 160 and $250 \mu \mathrm{m}$ data; green, GALEX far-UV; blue, radio $21 \mathrm{~cm}$ data. The star-forming disk is defined as the large circle. The small circles indicate individual dusty star-forming clumps, while small dotted ellipses indicate individual diffuse regions. b. Images of ESO 146-G14: red, the sum of Herschel 160 and $250 \mu \mathrm{m}$ data; green, GALEX far-UV; blue, radio $21 \mathrm{~cm}$ data. The disk is indicated as the large ellipse while individual star-forming clumps are shown as small circles.
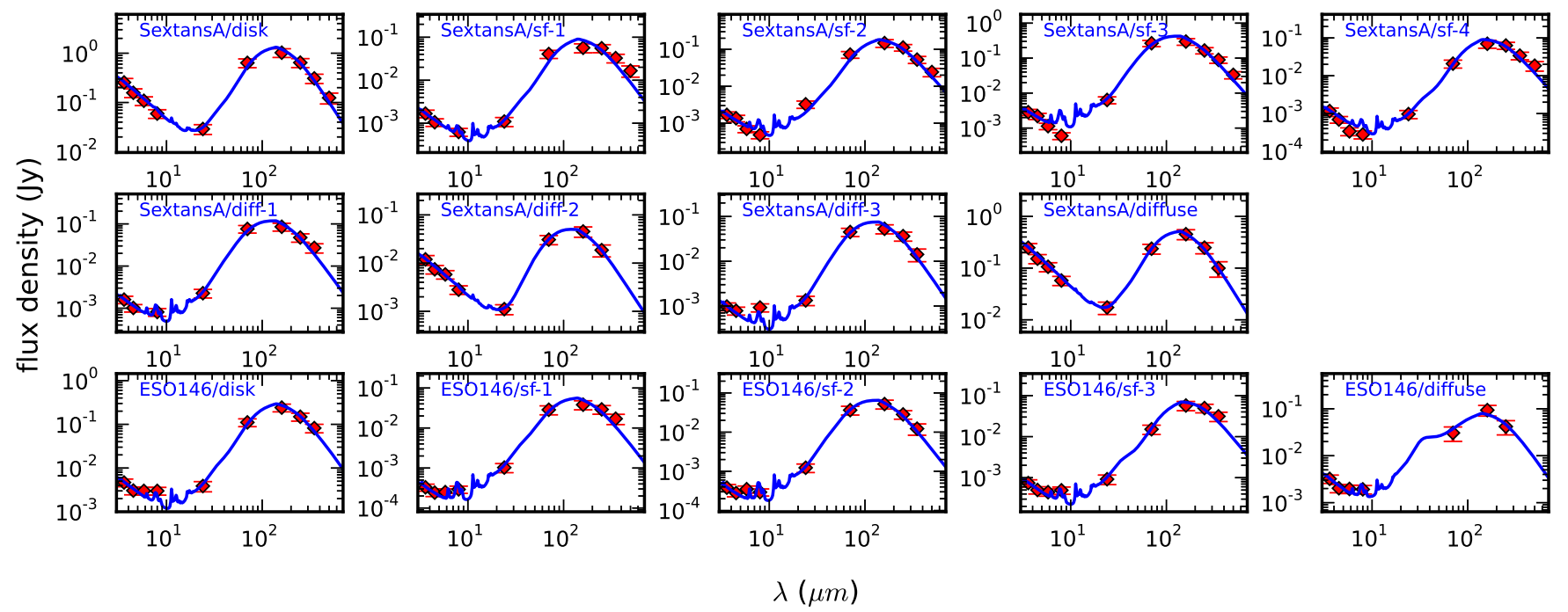

The blue solid line indicates the best-fit by the dust mode 20

Table 1: The Properties Of The Sample

\begin{tabular}{|c|c|c|c|c|c|c|c|}
\hline 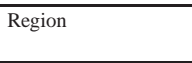 & $\begin{array}{l}\text { Right ascension } \\
\text { (J2000) }\end{array}$ & $\begin{array}{l}\begin{array}{l}\text { Declination } \\
\text { (J2000) }\end{array} \\
\end{array}$ & $\begin{array}{l}\mathrm{m}_{a}{ }^{*}, \mathrm{~m}_{b}{ }^{*} \\
(\operatorname{arcsec} ; \mathrm{kpc})\end{array}$ & $\begin{array}{l}\begin{array}{l}\text { Dust mass } \\
\left(\mathrm{M}_{\odot}\right)\end{array} \\
\end{array}$ & 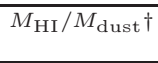 & $\begin{array}{l}\log \Sigma_{\mathrm{gas}^{\ddagger}} \\
\left(\log M_{\odot} / \mathrm{pc}^{2}\right)\end{array}$ & $\begin{array}{ll}\log \Sigma_{\mathrm{SFR}} \\
\left(\log M_{\odot} / \mathrm{yr}^{\prime} / \mathrm{kpc}^{2}\right)\end{array}$ \\
\hline SextansA/disk & 10:11:01.4 & $-04: 41: 25$ & $152 \times 152 ; 1.06 \times 1.06$ & $\left(9.5_{-1.0}^{+1.1}\right) \times 10^{3}$ & $\left(5.7_{-0.7}^{+0.6}\right) \times 10^{3}$ & \multirow{9}{*}{$\begin{array}{l}2.26_{-0.22}^{+0.23} \\
2.57_{-0.21}^{+0.21} \\
2.21_{-0.21}^{+0.23} \\
2.46_{-0.21}^{+0.21}\end{array}$} & \\
\hline SextansA/sf-1 & $10: 10: 56.9$ & $-04: 40: 27$ & $22 \times 22 ; 0.16 \times 0.16$ & $\left(9.9_{-1.5}^{+2.5}\right) \times 10^{2}$ & $\left(1.3_{-0.7}^{+0.6}\right) \times 10^{3}$ & & $-2.66 \pm 0.2$ \\
\hline SextansA/sf-2 & 10:11:10.0 & $-04: 41: 44$ & $22 \times 22 ; 0.16 \times 0.16$ & $\left(2 .{ }_{-0.2}^{+0.2}\right) \times 10^{3}$ & $\left(1.3_{-0.7}^{+0.6}\right) \times 10^{3}$ & & $-2.77 \pm 0.2$ \\
\hline SextansA/sf-3 & 10:11:06.2 & $-04: 42: 23$ & $32 \times 32 ; 0.22 \times 0.22$ & $\left(1.8_{-0.3}^{+0.4}\right) \times 10^{3}$ & $\left(3.2_{-0.7}^{+0.6}\right) \times 10^{3}$ & & $-2.32 \pm 0.2$ \\
\hline SextansA/sf-4 & $10: 10: 55.5$ & $-04: 42: 59$ & $22 \times 22 ; 0.16 \times 0.16$ & $\left(1.6_{-0.1}^{+0.1}\right) \times 10^{3}$ & $\left(4.1_{-6.6}^{+5.8}\right) \times 10^{2}$ & & \multirow[t]{6}{*}{$-3.19 \pm 0.2$} \\
\hline SextansA/diff-1 & $10: 10: 53.2$ & $-04: 41: 43$ & $38 \times 20 ; 0.26 \times 0.14$ & $\left(5.1_{-0.5}^{+0.8}\right) \times 10^{2}$ & $\left(6.9_{-0.7}^{+0.6}\right) \times 10^{3}$ & & \\
\hline SextansA/diff-2 & 10:11:09.2 & $-04: 41: 02$ & $21 \times 14 ; 0.15 \times 0.10$ & $\left(1.8_{-0.3}^{+0.3}\right) \times 10^{2}$ & $\left(8.6_{-0.7}^{+0.6}\right) \times 10^{3}$ & & \\
\hline SextansA/diff-3 & 10:10:54.0 & $-04: 40: 44$ & $27 \times 18 ; 0.19 \times 0.13$ & $\left(3.2_{-0.3}^{+0.6}\right) \times 10^{2}$ & $\left(6.6_{-0.7}^{+0.6}\right) \times 10^{3}$ & & \\
\hline SextansA/diffuse & & & & $\left(3.1_{-0.4}^{+0.3}\right) \times 10^{3}$ & $\left(1.4_{-0.1}^{+0.1}\right) \times 10^{4}$ & & \\
\hline ESO146-G14/disk & 22:13:01.3 & $-62: 04: 00$ & $90 \times 15 ; 9.34 \times 1.56$ & $\left(5.9_{-0.5}^{+0.9}\right) \times 10^{5}$ & $\left(2.5_{-0.5}^{+0.2}\right) \times 10^{3}$ & \multirow{5}{*}{$\begin{array}{l}1.21_{-0.22}^{+0.24} \\
1.12_{-0.22}^{+0.22} \\
1.77_{-0.21}^{+0.21}\end{array}$} & \\
\hline ESO146-G14/sf-1 & 22:13:06.0 & $-62: 03: 33$ & $10 \times 10 ; 1.04 \times 1.04$ & $\left(7.5_{-1.0}^{+2.1}\right) \times 10^{4}$ & $\left(1.6_{-0.5}^{+0.2}\right) \times 10^{3}$ & & $-3.46 \pm 0.2$ \\
\hline ESO146-G14/sf-2 & $22: 13: 02.5$ & $-62: 03: 52$ & $10 \times 10 ; 1.04 \times 1.04$ & $\left(6.2_{-0.8}^{+0.9}\right) \times 10^{4}$ & $\left(2.7_{-0.5}^{+0.2}\right) \times 10^{3}$ & & $-3.26 \pm 0.2$ \\
\hline ESO146-G14/sf-3 & 22:12:59.0 & $-62: 04: 14$ & $10 \times 10 ; 1.04 \times 1.04$ & $\left(2.7_{-0.2}^{+0.2}\right) \times 10^{5}$ & $(5 .+2.2) \times 10^{2}$ & & $-3.65 \pm 0.2$ \\
\hline ESO146-G14/diffuse & & & & $\left(2.5_{-0.3}^{+0.3}\right) \times 10^{5}$ & $\left(4.4_{-0.5}^{+0.2}\right) \times 10^{3}$ & & \\
\hline
\end{tabular}

* Major and minor axis lengths are given in arcsec and kpc.

${ }^{\dagger}$ The atomic gas to dust mass ratio. The atomic gas includes Helium by multiplying HI gas by a factor of 1.36 .

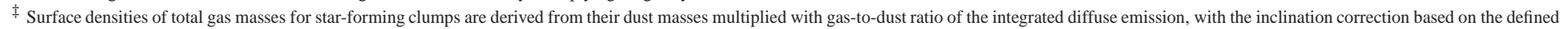
disk ellipse.

ฯ Surface densities of SFRs are derived from the combination of IR and far-UV tracer $\stackrel{23}{2}$, with the inclination corrected based on the defined disk ellipse. 
the same distribution as the SFRs of spiral galaxies at comparable gas densities.

As illustrated in Fig. 3, the derived dust-based gas masses of individual clumps are much higher than the atomic gas masses, indicating high molecular gas fractions. By subtracting the observed atomic gas from the derived dust-based gas mass for our seven starforming clumps, we find that the derived molecular gas mass is on average $\sim 6$ times larger than the atomic gas mass. For the star-forming clumps, the median and standard deviation of the molecular SFE is $\left.\log \left(\mathrm{SFE}_{\mathrm{H}_{2}}\left[\mathrm{yr}^{-1}\right]\right)\right)=-10.8 \pm 0.6$. The corresponding molecular gas depletion time in $\operatorname{logarithm}$ is $\log \left(\tau_{\mathrm{dep}}^{\mathrm{H}_{2}}[\mathrm{Gyr}]\right)=1.8 \pm 0.6$. This is much larger than the typical depletion time at sub-kpc scales of local spirals or dwarfs with oxygen abundance above $20 \%$ solar which is about $\log \left(\tau_{\text {dep }}^{\mathrm{H}_{2}}[\mathrm{Gyr}]\right)=0.3^{3 \sqrt{3}}$. Therefore star formation is strongly suppressed in the clumps, whether the star formation is scaled by total gas mass or by molecular gas mass alone.

Although model $\sqrt{1 / 2,4}$ of metallicity regulated star formation predict significantly reduced SFEs at our metallicities as illustrated in Fig. 3, the gas in the models is nevertheless mainly atomic, in contrast with our findings. The low SFE in the model is caused by a greatly reduced formation of molecular gas on the surface of dust grains 2 or possibly tied to enhanced heating of the atomic gas ${ }^{4}$. On the other hand, if we increase the depletion time of molecular gas in the models by an order of magnitude to $\sim 20 \mathrm{Gyr}$, the gas in the model is still mostly atomic at our observed gas densities. Studies 25 of HI dominated (by assuming low molecular gas fractions) dwarf galaxies suggest that their SFEs are not significantly lower than spiral galaxies. Our results extend these previous findings to lower metallicity, and suggest high molecular gas fractions do exist in star-forming clumps of extremely low metallicity.

The nature of this excess dust-based gas mass is still unclear. This gas is most likely in the molecular phase as the cold HI should be detected by $21 \mathrm{~cm}$ observations. If it is cold molecular gas, there should be associated CO emission. Extended Data Table 6 lists the predicted $\mathrm{CO}$ flux for these metal poor star-forming clumps by assuming the $\mathrm{CO}$ to- $\mathrm{H}_{2}$ factor, $\alpha_{\mathrm{CO}}=500 M_{\odot} \mathrm{pc}^{-2}\left(\mathrm{~K} \mathrm{~km} \mathrm{~s}^{-1}\right)^{-1}$, which is appropriate for regions of such low metallicity. It should be noted however, that there is large uncertainty in this value ${ }^{6}$. Region "sf-3" in Sextans A does indeed have mm-wave observations 26 . Accounting for a filling factor of one third of dust emission in the $\mathrm{CO}$ beam, we estimate a $3-\sigma$ upper limit to the CO flux that is about a factor of three above our predicted value. Therefore much deeper mm observations would be needed to detect the $\mathrm{CO}$ emission from the excess cold molecular gas in Sextans A. It is unclear what mechanisms prevents this abundant molecular gas from forming new stars. It is possible that the molecular gas does not effectively cool due to intense radiation fields, slowing the SFRs in these environments. Warm $\mathrm{H}_{2}$ gas with surface densities as high as $50 \mathrm{M}_{\odot} / \mathrm{pc}^{2}$ is seen in some blue compact dwarfs $\sqrt{27}$. Although our two galaxies are not blue compact dwarfs, the SFR surface densities of the star-forming regions in our galaxies are comparable to those found in blue compact dwarfs. This similarity suggests the possible presence of abundant warm $\mathrm{H}_{2}$ in our two extremely metal poor galaxies. The Extended Data Table 6 also lists the predicted $\mathrm{H}_{2} S(1) 17.03$ $\mu \mathrm{m}$ line flux based on the example of Mrk 996 $6^{27}$. There is archived Spitzer spectroscopic observations of the region "sf-3" of Sextans A. Based on the archived reduced data, after accounting for the difference between the Spitzer aperture and the size of our "sf-3", the observed $\mathrm{H}_{2} 17.03 \mu \mathrm{m}$ flux is about $4 \times 10^{-16} \mathrm{~W} / \mathrm{m}^{2}$, a factor of two lower than our predicted value.

The extremely metal poor galaxies may provide a close-up view of the highly inefficient star formation occurring in galaxies in the early Universe where Population II stars form out of gas whose metallicity is $1 / 10$ Solar or less 14 . The suppressed SFEs in extremely low metallicity galaxies at early epochs may be able to reconcile some tensions be-

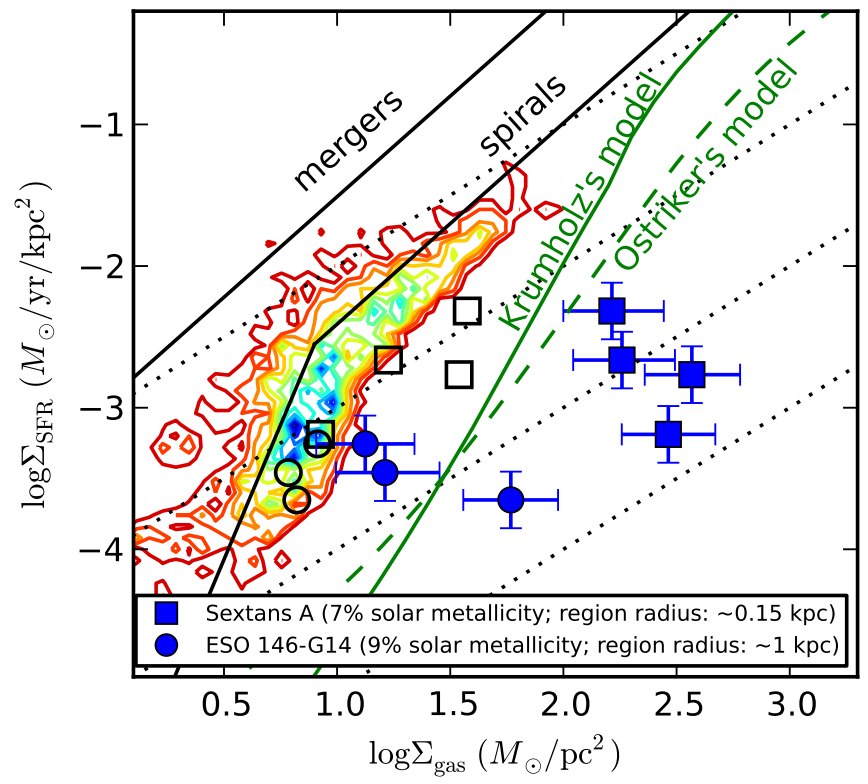

Figure 3 | Seven metal poor star-forming clumps show extremely low star formation efficiencies: Our data with 1- $\sigma$ errors (filled symbols for dust-based total gas mass while open symbols for atomic gas only), were compared to spiral disks at sub-kpc scales 3 (color contour), and integrated spiral and mergers 24 (black lines) in the plane of the SFR vs. gas surface densities. The green solid and dashed lines represent predictions of the mode ${ }^{2}$ at $8 \%$ solar metallicity and a clumping factor of 1 , and mode 4 at $8 \%$ solar metallicity. Dotted lines indicate constant SFEs of, from the top to the bottom, $10^{-9,-10,-11,-12} \mathrm{yr}^{-1}$.

tween observations and theoretical models for early galaxy evolution 28

1. Ostriker, E. C. McKee, C. F., Leroy, A. K. Regulation of star formation rates in multiphase galactic disks: a thermal/dynamical equilibrium model. Astrophys. J. 721, 975-994 (2010).

2. Krumholz, M R. The star formation law in molecule-poor galaxies. Mon Not. R. Astron. Soc. 436, 2747-2762 (2013).

3. Bigiel et al. The star formation law in nearby galaxies on sub-kpc scales. Astron. J. 136, 2846-2871 (2008).

4. Bolatto, A. D. et al. The state of the gas and the relation between gas and star formation at low metallicity: the small magellanic cloud. Astrophys. J. 741, 12-30 (2011).

5. Elmegreen, B. G. et al. Carbon monoxide in clouds at low metallicity in the dwarf irregular galaxy WLM. Nature 495, 487-489 (2013).

6. Bolatto, A. et al. The CO-to-H2 Conversion Factor. Annu. Rev. Astron. Astrophys. 51, 207-268 (2013).

7. Leroy, A. K. et al. The CO-to-H2 conversion factor from infrared dust emission across the Local Group. Astrophys. J. 737, 12-24 (2011).

8. Fisher, D.et al The rarity of dust in metal-poor galaxies. Nature 505, 186-189 (2014).

9. Hunt, L, K.et al ALMA observations of cool dust in a low-metallicity starburst, SBS 0335-052. Astron. Astrophys. 561, A49 (2014).

10. Remy-Ruyer, A.et al Gas-to-dust mass ratios in local galaxies over a 2 dex metallicity range. Astron. Astrophys. 563, A31 (2014).

11. Pettini, M., Pagel, B. [OIII]/[NII] as an abundance indicator at high redshift. Mon. Not. R. Astron. Soc. 348, L59-L63 (2004).

12. Kniazev, A. Y. et al. Spectrophotometry of Sextans A and B: Chemical Abundances of $\mathrm{H}$ II Regions and Planetary Nebulae. Astron. J. 130, 1558-1573 (2005).

13. Bergvall, N., Ronnback, J. ESO 146-G14 A retarded disc galaxy. Mon. Not. R. Astron. Soc. 273, 603-614 (1995).

14. Wise, J. et al The Birth of a Galaxy: Primordial Metal Enrichment and Stellar Populations. Astrophys. J. 745, 50-59 (2012).

15. Sandstrom, K. M.et al The CO-to-H2 Conversion Factor and Dust-to-gas Ratio on Kiloparsec Scales in Nearby Galaxies. Astrophys. J. 777, 5-37 (2013).

16. Poglitsch, et al. The Photodetector Array Camera and Spectrometer (PACS) on the Herschel Space Observatory. Astron. Astrophys. 518, L2 (2010).

17. Griffin, M. J. et al. The Herschel-SPIRE instrument and its in-flight performance. Astron. Astrophys. 518, L3 (2010). 
18. Ott, J. et al. VLA-ANGST: A high-resolution HI Survey of Nearby Dwarf Galaxies. Astron. J. 144, 123-195 (2012).

19. Peters S. P. C. et al. The Shape of Dark Matter Halos in Edge-on Galaxies: I. Overview of $\mathrm{HI}$ observations. Preprint at http://arxiv.org/abs/1303.2463 (2013).

20. Draine, B. T., Li, A. Infrared emission from interstellar dust. IV. the silicate-graphite-pah model in the post-Spitzer era. Astrophys. J. 657, 810-837 (2007).

21. Draine, B. T.et al Andromeda's Dust. Astrophys. J. 780, 172-189 (2014).

22. Westmoquette, M. S.et al Piecing together the puzzle of NGC 5253: abundances, kinematics and WR stars. Astron. Astrophys. 550, 88-103 (2013).

23. Leroy, A.et al The Star Formation Efficiency in Nearby Galaxies: Measuring Where Gas Forms Stars Effectively. Astrophys. J. 136, 2782-2845 (2008).

24. Daddi, E. et al. Different Star Formation Laws for Disks Versus Starbursts at Low and High Redshifts. Astrophys. J. 714, 118-122 (2010).

25. Cormier, D. et al. The molecular gas reservoir of 6 low-metallicity galaxies from the Herschel Dwarf Galaxy Survey. A ground-based follow-up survey of $\mathrm{CO}(1-0), \mathrm{CO}(2-1)$, and $\mathrm{CO}(3-2)$. Astron. Astrophys. 564, A121 (2014).

26. Taylor, C. L., Kobulnicky, H. A., Skillman, E. D. CO Emission in LowLuminosity, H I-rich Galaxies. Astron. J. 116, 2746-2756 (1998).

27. Hunt, L. et al. THE SPITZER VIEW OF LOW-METALLICITY STAR FORMATION. III. FINE-STRUCTURE LINES, AROMATIC FEATURES, AND MOLECULES. Astrophys. J. 712, 164-187 (2010).

28. Kuhlen, M. et al. Dwarf galaxy formation with $\mathrm{H} 2$-regulated star formation. Astrophys. J. 749, 36-57 (2012).

Acknowledgements Y.S. acknowledges support for this work from Natura Science Foundation of China (NSFC) under the grant of 11373021, the Strategic Priority Research Program "The Emergence of Cosmological Structures" of the Chinese Academy of Sciences (CAS), Grant No. XDB09000000, and Nanjing University 985 grant. Y.G. acknowledges support from NSFC grants (11173059 and 11390373) and CAS Program (Grant No. XDB09000000). J.W. is supported by National 973 program (2012CB821805) and NSFC grant (11173013). Z.Z. acknowledges support from the European Research Council (ERC) in the form of Advanced Grant, CosmicisM. Q.G. is supported under the NSFC grants (11273015 and 11133001) and the National 973 program (2013CB834905). We are grateful to F. Bigiel for making their data points available to plot contours in Figure 3, S. P. C. Peters for making available their $\mathrm{HI}$ gas map of ESO 146-G14 to us, and L. Piazzo for help in Herschel data reduction. Herschel is an ESA space observatory with science instruments provided by European-led Principal Investigator consortia and with important participation from NASA. This work was supported in part by the Spitzer Space Telescope, which is operated by the Jet Propulsion Laboratory, California Institute of Technology under a contract with NASA. It was also supported in part by a NASA Herschel grant (OT2_yshi_3) issued by JPL/Caltech.

Author Contributions Y.S. led the Herschel proposal, Herschel data reduction and writing of the manuscript. L.A. help develop Herschel observations and writing of the manuscript. G.H. and S.S. assisted in the Herschel proposal. All authors discussed and commented on the manuscript.

Author Information Reprints and permissions information is available at www.nature.com/reprints. The authors declare no competing financial interests. Readers are welcome to comment on the online version of the paper. Correspondence and requests for materials should be addressed to YS (yshipku@gmail.com). 


\section{METHODS}

\section{Observations and data reduction}

Herschel infrared images were generated through scan map modes at 70 and $160 \mu \mathrm{m}$ with PACS, and small map modes at 250, 350, and $500 \mu \mathrm{m}$ with SPIRE (PI: Y. Shi, PID: OT2_yshi_3). The half power beam widths at these wavelengths are about $5^{\prime \prime}, 12^{\prime \prime}, 20^{\prime \prime}, 28^{\prime \prime}$ and $39^{\prime \prime}$, respectively. The mapping field size at each band is set to be at least 1.5 times the optical size $D_{25}$ of the galaxy where $D_{25}$ is the $B$ band isophote at $25 \mathrm{mag}$ arcsec ${ }^{-2}$, which is large enough to provide blank sky for sky removal. Excluding overheads, the effective integration times per sky position in two PACS bands are $1.9 \mathrm{hr}$ and $1.6 \mathrm{hr}$ for Sextans A and ESO 146-G14, respectively, and in three SPIRE bands is 6 min for the two targets. For Sextans A at $160 \mu \mathrm{m}$, additional Herschel archived data (PI: D. Hunter) with similar exposure time to ours were further combined with our own observations. The data reduction was performed with unimap version $5.5^{\frac{29[30]}{2}}$ with procedures basically following the standard one. To better recover the extended emission, the GLS map maker starts with the zero map and the length of median filter for the PGLS algorithm is set to be twice the default value $(=60)$. Unimap is a Matlab-based Herschel data processing software. Unimap takes as input the level 1 pipeline data as produced by Herschel Standard Product Generator (Version 11.1.0 for this work) and identifies signals in time ordered pixels. After removing glitch and drift, the final maps were made with pixel scales of $1^{\prime \prime}, 2^{\prime \prime}, 4^{\prime \prime}, 6^{\prime \prime}$ and $8^{\prime \prime}$ at 70,160 , 250,350 and $500 \mu \mathrm{m}$, respectively.

The reduced far-UV images were obtained from the GALEX data archive hosted by the Multi-Mission archive at the Space Telescope Science Institute. GALEX has a spatial resolution about $5^{\prime \prime}$ in the farUV 31 . The exposure times for Sextans A and ESO146-G14 are 1698 $\mathrm{sec}$ and $111 \mathrm{sec}$, respectively. Sextans A has reduced Spitzer images at 3.6, 4.5, 5.8, 8.0, 24, 70 and $160 \mu \mathrm{m}$ obtained by the LVL program 32 available in the NASA infrared science archive. The corresponding spatial resolutions are about $5^{\prime \prime} \times \lambda / 24 \mu \mathrm{m}$. For ESO146-G14, the data at 3.6, 4.5, 5.8, 8.0 and $24 \mu \mathrm{m}$ were available in the Spitzer Heritage Archive at the Spitzer Science Center and the archived enhanced imaging products were used. The Spitzer 70 and $160 \mu \mathrm{m}$ integrated fluxes ${ }^{33}$ of ESO 146-G14 were used to compared to our PACS measurements

\section{Infrared flux measurements}

Our Herschel flux measurements start with aperture definitions followed by sky subtractions. As shown in Extended Data Fig. 1, the aperture of the star-forming disk of each galaxy is defined as an ellipse to closely follow the $10 \sigma$ contour above the sky of the far-UV image, corresponding to surface brightness levels of 25.9 and $26.2 \mathrm{mag} \mathrm{arcsec}^{-2}$ (AB magnitude) for Sextans A and ESO 146-G14, respectively. The results of this study change little if 5- $\sigma$ or $20-\sigma$ contours were used to define the disk aperture. Within each star-forming disk, star-forming clumps are defined as circle regions showing both elevated $(>3-\sigma)$ far-UV and IR emission at $160 \mu \mathrm{m}$ after convolving two images to resolutions at PACS $350 \mu \mathrm{m}$ ( 28 arcsec). Here the $\sigma$ is the standard deviation of pixel values within the star-forming disk. The clump radius is listed in Extended Data Table 1. For Sextans A, we also identified three individual diffuse regions that are below 3- $\sigma$ local backgrounds but show extended emission at 70 and $160 \mu \mathrm{m}$ resolutions. Within the disk, IR point sources that do not have corresponding far-UV counterparts are identified as background sources rather than star-forming regions in the disk, since none of the identified clumps has IR/far-UV flux ratios smaller than about 0.2 .

The sky annuli were defined to be between 1.1 times and 1.5 times the disk aperture. The mode of the sky pixel brightness distribution is subtracted from the image in each case. However, since faint, undetected background sources can make the noise distribution non-
Gaussian, we also test the validity of our results by subtracting off the mean value of the sky after masking out bright sources in the sky annuli.

In total, we derive three types of flux measurements for each region as listed in Extended Data Table 1. For the first, referred to as m1, we use the mode of the sky brightness after masking off all potential background sources in the disk. For the second, referred to at $m 2$, we again subtract off the mode of the sky distribution, but treat the suspected background sources as embedded star-formation regions in the disk. For the third, referred to as $\mathrm{m} 3$, we first mask out all potential background sources, then subtract the mean of the sky pixels. All potential background sources and bright sources within the sky annuli are identified through sextractor ${ }^{\sqrt{34}}$ with further visual checks. We use the first flux estimates, $\mathrm{m} 1$, as the fiducial for the analysis presented here.

For images at each wavelength, aperture photometry was performed after subtracting the sky background. The aperture corrections were further applied at each wavelength based on the corresponding point spread function at that wavelength. Diffuse emission within the disk is measured as the residual after subtracting the flux from all identified star-forming clumps. The result is presented in Extended Data Table 1 where each region has three measurements detailed above, referred as $\mathrm{m} 1, \mathrm{~m} 2$ and $\mathrm{m} 3$ measurements.

The flux measurements in the Spitzer band were carried out in a similar way to the Herschel m-1 method and the result is listed in Extended Data Table 2.

\section{Infrared flux uncertainty estimates}

The Herschel flux uncertainties are given by the following formula

$\sigma=\left(\sigma_{\text {photon, confusion }}^{2} \times A_{\text {region }}+\frac{\sigma_{\text {photon,confusion }}^{2}}{A_{\text {sky }}} A_{\text {region }}^{2}+\sigma_{\mathrm{PSF}-\text { offset }}^{2}\right)+\sigma_{\text {abs }- \text { ca }}^{2}$

, where $A_{\text {region }}$ and $A_{\text {sky }}$ are the area of target regions and sky annuli, respectively. $\sigma_{\text {photon,confusion }}$ is the scatter of the sky pixel brightness distribution. Extended Data Table 3 compares our measured $\sigma_{\text {photon,confusion }}$ to the predicted photon and confusion noises estimated using the Herschel Observing Tool (HSPOT) for our targets. The noise in our images is consistent with the quadratic sum of the HSPOT photon and confusion noise to within a factor of two. The second term in the above equation gives the scatter of the derived sky brightness. $\sigma_{\mathrm{PSF}-\text { offset }}$ is the flux uncertainty caused by the accuracy in positioning an aperture onto a given star-forming clump. For each star-forming region, we estimated this by randomly offsetting the peak of a modelled PSF to $1 / 2$ the Nyquist sampled beam and measuring the flux variation within the given source aperture. The final term is the absolute flux calibration error taken to be $20 \%$ across all wavelengths based on the PACS and SPIRE instrument handbooks as well as systematic comparisonss $\stackrel{\sqrt{35-38}}{ }$ between PACS and MIPS measurement. Extended Data Table 1 listed the quadratic sum of the first three errors. The final error term is added in quadrature when doing the SED fitting but not used in Extended Data Table 1 as it is a systematic error of the Herschel Space Telescope. Our estimated errors are quite reasonable compared to the expected point source flux errors from HSPOT (also listed in Extended Table 3). Note that although in the SPIRE bands the confusion noise is 2-3 times higher than the photon noise, this can be mitigated by using a PACS 160 -micron prior on the position ${ }^{39}$. We can further compare our noise estimates to those from other Herschel observations of similar depth. For example, the Herschel lensing survey ${ }^{40}$ reported a $1-\sigma$ point-source depth of $2.4 \mathrm{mJy}$ at $250 \mu \mathrm{m}$ and $3.4 \mathrm{mJy}$ at $350 \mu \mathrm{m}$ for on-source exposure per sky position of $36 \mathrm{mins}$ with position priors from short wavelengths, compared to our 2-4 mJy at 250 $\mu \mathrm{m}$ and 3-5 mJy at $250 \mu \mathrm{m}$ for our 6-10 mins on source exposures.

We further carried out additional checks on the measured flux by 
comparing Spitzer and Herschel photometry. For Sextans A, we found that individual star-forming clumps as well as the diffuse region have $70 \mu \mathrm{m}$ Herschel fluxes consistent with Spitzer measurements within $30 \%$. And the integrated light of Sextans A and ESO146-G14 at both 70 and $160 \mu \mathrm{m}$ are also consistent within $30 \%$ between the Spitzer and Herschel data-sets.

To check the possibility that the diffuse emission is due to the background fluctuations or not. We randomly position the source aperture over the observed field of view, and then compared the measured fluxes to the quoted error of the target diffuse emission. For ESO 146-G14, we can randomly position about 30 apertures and found that none of them have $\mathrm{S} / \mathrm{N}$ larger than 3 at bands where the diffuse emission is detected. For Sextans A, the observed field of view is not large enough for us to perform similar exercises.

\section{IR SED fitting and dust mass measurements}

We fit the infrared data with the dust model ${ }^{20}$ in order to estimate the dust mass of each region. As shown above, we have three types of flux measurements, and fit all three with the dust model. We choose a Milky Way grain size distribution ${ }^{20}$ and fix the PAH fraction to the minimum (the total dust mass that is in PAHs $q_{\mathrm{PAH}}=0.47 \%$ ) given the low metallicity (the result does not change if this parameter is set free). To further check the effect of different dust grains, for the first type of flux measurements (m1), SMC and LMC dust grains that have different grain compositions and size distributions are also explored. Overall we thus have five dust mass measurements for each region, three of them are for different types of flux measurements with Milky Way grains (referred as m1-MW, m2-MW, m3-MW), two are for two different grain size distributions fitted to the first type of flux measurements (m1-SMC and $\mathrm{m1}$-LMC).

In the following we take the m1-MW as an example to illustrate the fitting procedure. The results are plotted in Fig. 2 and listed in Extended Data Table 4. To do the fit, a $4000 \mathrm{~K}$ black-body spectrum was first added to represent the emission from stellar photospheres which dominates at $<10 \mu \mathrm{m}$. The model was then left with four free parameters, including the dust mass, the minimum $\left(U_{\min }\right)$ and maximum intensity $\left(U_{\max }\right)$ of the stellar radiation field that is responsible for heating the dust and the fraction $(1-\gamma)$ of dust exposed to the minimum starlight intensity (i.e. $U_{\min }$ ). Similar to studies of dust emission in spirals $\frac{15}{15}, U_{\max }$ was further fixed to the maximum of $10^{6}$. We then performed SED fitting with three free parameters for Sextans A and ESO 146-G14.

As listed in Extended Data Table 4, the reduced $\chi^{2}$ for the majority of the fits have values of around unity, while sf- 1 , sf- 2 and diff- 3 of Sextans-A have reduced $\chi^{2}$ around 10. As shown in Fig. 2, the $160 \mu \mathrm{m}$ photometry of sf- 1 and diff- 3 shows large deviations from the best fit, while 24 and $70 \mu \mathrm{m}$ photometry of sf-b deviates largely from the best fit. Uncertainties in the derived dust mass were estimated by performing 100 fits to each source after adding in Gaussian noise.

We also carried out simple modified black-body fitting to IR SEDs of Sextans A and ESO 146-G14 that have enough far-IR photometric data points. As listed in Extended Data Table 4, the dust temperature of star-forming clumps is between 30 and $50 \mathrm{~K}$ while the dust in the diffuse region is about $30-40 \mathrm{~K}$.

\section{Measurements of SFR and gas mass surface den- sities}

The SFRs of star-forming clumps were measured by combining the FUV and 24-micron data, which represent the unobscured and obscured star-formation, respectively 23 . The derived SFRs are uniformly assigned a 0.2 dex error to account for the systematic errors in deriving SFRs from UV and IR photometry (the photon noise is comparatively small). The SFR surface density is further corrected for the inclination based on the defined disk ellipsity. The final result is listed in Table 1.

With derived dust masses, we estimated the GDR of the diffuse region as the ratio of atomic to dust mass. The GDR of the integrated diffuse region is then applied to individual star-forming clumps to derive the total gas mass and thus the gas mass surface density $\left(\Sigma_{\text {gas }}\right)$. Extended Data Table 5 lists the result for five fits that are m1-MW, m2MW, m3-MW, m1-SMC and m1-LMC. The associated uncertainties of $\Sigma_{\text {gas }}$ are the quadratic sum of errors of dust mass measurements, errors of GDRs of diffuse regions contributed by uncertainties on HI and dust mass estimates of diffuse regions, 0.2 dex for the GDR variation across the disk based on studies of spiral galaxies ${ }^{15}$. The result of the m1-MW fit is used as a fiducial, as listed in Table 1 and shown in Fig. 3. Our conclusions of significantly reduced SFEs in seven metal poor star-forming clumps change little if adopting other fitting results in Extended Data Table 5. In addition, there are some concerns that the PACS may miss some extended emission, although this is not seen by our comparisons to Spitzer and in investigations by others 35.38 . To test this effect, we artifically increased the PACS fluxes of the diffuse emission by $30 \%$ while keeping the SPIRE fluxes as they were, the resulting surface densities of gas masses of star-forming clumps drop by no more than 0.1 dex. In additiona, three individual diffuse regions of Sextans A has similar GDR, only a factor of 1.5-2 lower than that of the integrated diffuse emission, indicating that our GDR estimate is reasonable.

We investigate if the derived $\Sigma_{\text {gas }}$ can be significantly lowered by forcing changes in dust model parameters, specifically raising $U_{\min }$ which can result in lower dust masses and hence lower gas surface densities and higher star formation efficiencies. In the following discussion, we take the m1-MW fit as the case study. For both targets, the best-fit $U_{\min }$ of all regions are relatively small. We thus keep the best-fit $U_{\min }$ for the diffuse region but gradually increase $U_{\min }$ of starforming clumps to decrease their $\Sigma_{\text {gas }}$. We find that the star-forming clumps in Sextans A can move into the spiral galaxy regime of Fig. 3 if the $\mathrm{U}_{\min }$ rises above 20 . However, in this case the corresponding chisquared rises to 40-60. For star-forming clumps in ESO 146-G14, the $U_{\text {min }}$ needs to be larger than 15 to move into the spiral regime however these fits are again poor, with chi-squared values of 10-30. Therefore the significantly reduced SFEs of star-forming clumps in Sextans A and ESO 146-G14 should be robust to the change in their $U_{\text {min }}$.

29. Traficante, A. et al. Data reduction pipeline for the Hi-GAL survey. Maris 416, 2932-2943 (2011).

30. Piazzo, L. et al. Artifact removal for GD'S map makers by means of postprocessing. WEER Trans. on Image Processing 21, 3687-36 (2012).

31. Morrissey, P. et al. The Calibration and Data Products of GALEX. Astrophys. J. Supp. 173, 682-697 (2007)

32. Dale D. A. et al. The Spitzer Local Volume Legacy: survey description and infrared photometry. Astrophys. J. 703, 517-556 (2009).

33. Engelbracht, C. W. et al Metallicity Effects on Dust Properties in Starbursting Galaxies. Astrophys. J. 678, 804-827 (2008).

34. Bertin, E. et al. SExtractor: Software for source extraction. A\&AS 117, 393-404 (1996).

35. Sauvage, M. Experiments in photometric measurements of extended sources http://herschel.esac.esa.int/twiki/pub/Public/PacsCalibrationWeb/ExtSrcPhotom.pdf (2011).

36. Ali, B. Surface brightness comparison of PACS blue array with IRAS and Spitzer/MIPS images. https://nhscsci.ipac.caltech.edu/pacs/docs/Photometer/PICC-NHSCTN-029.pdf (2011)

37. Paladini, R. et al Assessment analysis of the extended emission calibration for the PACS red channel. https://nhscsci.ipac.caltech.edu/pacs/docs/Photometer/PICC-NHSCTR-034.pdf (2012)

38. Paladini, R. et al PACS Map-making Tools: Analysis and Benchmarking. http://herschel.esac.esa.int/twiki/pub/Public/PacsCalibrationWeb/pacs_mapmaking_repc (2013). 
39. Elbaz, D. et al. GOODS-Herschel: an infrared main sequence for starforming galaxies. Astron. Astrophys. 533, 119-144 (2011).

40. Egami, E. et al. The Herschel Lensing Survey (HLS): Overview. Astron. Astrophys. 518, 12-16 (2010). 


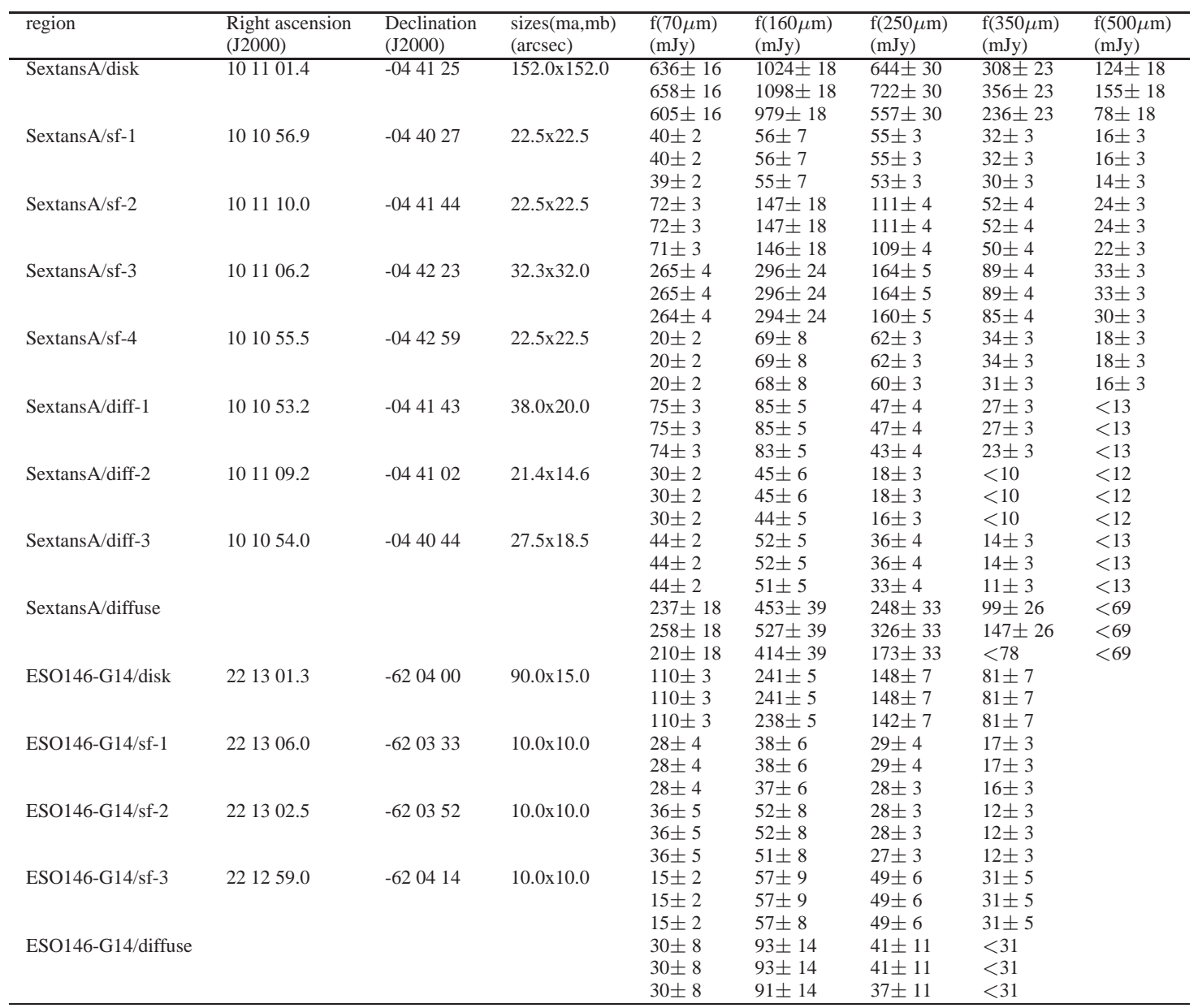

Extended Data Table $1 \mid$ PACS and SPIRE photometry for the selected regions: For each region, we give three types of flux measurements (referred as $\mathrm{m} 1$, m2 and $\mathrm{m} 3$ in the text). The 1- $\sigma$ flux errors include photon and confusion noise, scatter of the sky brightness, and uncertainties in the flux due to mis-centering of extraction apertures. The 3- $\sigma$ upper limits are given where appropriate. The uncertainties in the absolute flux calibration are not included here, but are added in quadrature before performing the SED fitting as described in the text.

\begin{tabular}{llllll}
\hline region & $\begin{array}{l}\mathrm{f}(3.6 \mu \mathrm{m}) \\
(\mathrm{mJy})\end{array}$ & $\begin{array}{l}\mathrm{f}(4.5 \mu \mathrm{m}) \\
(\mathrm{mJy})\end{array}$ & $\begin{array}{l}\mathrm{f}(5.8 \mu \mathrm{m}) \\
(\mathrm{mJy})\end{array}$ & $\begin{array}{l}\mathrm{f}(8.0 \mu \mathrm{m}) \\
(\mathrm{mJy})\end{array}$ & $\begin{array}{l}\mathrm{f}(24 \mu \mathrm{m}) \\
(\mathrm{mJy})\end{array}$ \\
\hline SextansA/disk & $255.33 \pm 0.06$ & $157.29 \pm 0.05$ & $108.28 \pm 0.26$ & $59.46 \pm 0.23$ & $28.98 \pm 3.00$ \\
SextansA/sf-1 & $1.67 \pm 0.01$ & $1.05 \pm 0.01$ & $<0.12$ & $0.62 \pm 0.04$ & $1.09 \pm 0.14$ \\
SextansA/sf-2 & $1.68 \pm 0.01$ & $1.36 \pm 0.01$ & $0.70 \pm 0.04$ & $0.48 \pm 0.04$ & $3.25 \pm 0.34$ \\
SextansA/sf-3 & $2.85 \pm 0.01$ & $2.20 \pm 0.01$ & $1.14 \pm 0.06$ & $0.60 \pm 0.05$ & $6.36 \pm 0.65$ \\
SextansA/sf-4 & $1.17 \pm 0.01$ & $0.69 \pm 0.01$ & $0.34 \pm 0.04$ & $0.28 \pm 0.04$ & $0.97 \pm 0.13$ \\
SextansA/diff-1 & $1.60 \pm 0.01$ & $1.01 \pm 0.01$ & $<0.15$ & $0.81 \pm 0.04$ & $2.27 \pm 0.25$ \\
SextansA/diff-2 & $11.96 \pm 0.01$ & $7.43 \pm 0.01$ & $5.83 \pm 0.03$ & $2.81 \pm 0.03$ & $1.11 \pm 0.13$ \\
SextansA/diff-3 & $0.98 \pm 0.01$ & $0.78 \pm 0.01$ & $<0.12$ & $0.93 \pm 0.04$ & $1.33 \pm 0.16$ \\
SextansA/diffuse & $247.96 \pm 0.06$ & $151.99 \pm 0.05$ & $106.00 \pm 0.29$ & $57.47 \pm 0.26$ & $17.31 \pm 3.11$ \\
ESO146-G14/disk & $4.67 \pm 0.01$ & $3.05 \pm 0.02$ & $3.00 \pm 0.08$ & $3.00 \pm 0.08$ & $3.87 \pm 0.66$ \\
ESO146-G14/sf-1 & $0.33 \pm 0.00$ & $0.24 \pm 0.00$ & $0.25 \pm 0.02$ & $0.29 \pm 0.02$ & $1.03 \pm 0.16$ \\
ESO146-G14/sf-2 & $0.38 \pm 0.00$ & $0.28 \pm 0.00$ & $0.35 \pm 0.02$ & $0.29 \pm 0.02$ & $1.23 \pm 0.18$ \\
ESO146-G14/sf-3 & $0.74 \pm 0.00$ & $0.50 \pm 0.00$ & $0.44 \pm 0.02$ & $0.49 \pm 0.02$ & $0.92 \pm 0.16$ \\
ESO146-G14/diffuse & $3.22 \pm 0.01$ & $2.03 \pm 0.02$ & $1.96 \pm 0.08$ & $1.92 \pm 0.08$ & $<2.17$ \\
\hline
\end{tabular}

Extended Data Table 2 | Spitzer photometry: The Spitzer photometric measurements were performed in a similar way to the Herschel m1 method.

\begin{tabular}{|c|c|c|c|c|c|}
\hline \multirow[t]{3}{*}{ galaxy/band } & \multicolumn{3}{|c|}{ Extended Source } & \multicolumn{2}{|c|}{ Point Sources } \\
\hline & $\sigma_{\text {measured-sky }}$ & $\sigma_{\mathrm{HSPOT}}$,photon & $\sigma_{\text {HSPOT }}$, confusion & $\sigma_{\mathrm{HSPOT}}$, photon & $\sigma_{\text {HSPOT }}$,confusion \\
\hline & $(\mathrm{MJy} / \mathrm{sr})$ & $(\mathrm{MJy} / \mathrm{sr})$ & $(\mathrm{MJy} / \mathrm{sr})$ & (mJy) & (mJy) \\
\hline SextansA/70 $\mu \mathrm{m}$ & 2.86 & 2.03 & 0.22 & 0.52 & 0.08 \\
\hline SextansA/160 $\mu \mathrm{m}$ & 1.20 & 0.92 & 0.74 & 0.83 & 1.34 \\
\hline Sextans $\mathrm{A} / 250 \mu \mathrm{m}$ & 0.93 & 0.24 & 1.19 & 2.86 & 7.0 \\
\hline Sextans $\mathrm{A} / 350 \mu \mathrm{m}$ & 0.49 & 0.11 & 0.67 & 2.38 & 8.2 \\
\hline ESO146-G14/70 $\mu \mathrm{m}$ & 1.82 & 1.53 & 0.20 & 0.60 & 0.08 \\
\hline ESO146-G14/160 $\mu \mathrm{m}$ & 1.10 & 0.99 & 0.74 & 1.33 & 1.33 \\
\hline ESO146-G14/250 $\mu \mathrm{m}$ & 0.75 & 0.24 & 1.18 & 2.86 & 7.0 \\
\hline ESO146-G14/350 $\mu \mathrm{m}$ & 0.46 & 0.11 & 0.67 & 2.38 & 8.1 \\
\hline
\end{tabular}

Extended Data Table 3 | Measured sky noises of our observations compared to predictions by HSPOT: HSPOT stands for the Herschel Observation planning tool. 


\begin{tabular}{|c|c|c|c|c|c|c|c|}
\hline region & $\mathrm{U}_{\min }$ & $\mathrm{U}_{\max }($ fixed) & $\gamma$ & $\chi^{2} /$ dof & $\begin{array}{l}M_{\text {dust }} \\
\left(\mathrm{M}_{\odot}\right)\end{array}$ & $M_{\mathrm{HI}} / M_{\text {dust }}$ & $\begin{array}{l}T_{\text {dust }} \\
(\mathrm{K})\end{array}$ \\
\hline SextansA/disk & 2.0 & $10^{6}$ & 0.01 & 1.31 & $\left(9.5_{-1.0}^{+1.1}\right) \times 10^{3}$ & $\left(5.7_{-0.7}^{+0.6}\right) \times 10^{3}$ & $33 \pm 1$ \\
\hline SextansA/sf-1 & 1.2 & $10^{6}$ & 0.00 & 9.00 & $\left(9.9_{-1.5}^{+2.5}\right) \times 10^{2}$ & $\left(1.3_{-0.7}^{+0.6}\right) \times 10^{3}$ & $45 \pm 7$ \\
\hline SextansA/sf-2 & 1.2 & $10^{6}$ & 0.00 & 14.41 & $\left(2 ._{-0.2}^{+0.2}\right) \times 10^{3}$ & $\left(1.3_{-0.7}^{+0.6}\right) \times 10^{3}$ & $28 \pm 2$ \\
\hline SextansA/sf-3 & 4.0 & $10^{6}$ & 0.00 & 2.87 & $\left(1.8_{-0.3}^{+0.4}\right) \times 10^{3}$ & $\left(3.2_{-0.7}^{+0.6}\right) \times 10^{3}$ & $38 \pm 3$ \\
\hline SextansA/sf-4 & 0.7 & $10^{6}$ & 0.00 & 2.21 & $\left(1.6_{-0.1}^{+0.1}\right) \times 10^{3}$ & $\left(4.1_{-6.6}^{+5.8}\right) \times 10^{2}$ & $27 \pm 2$ \\
\hline SextansA/diff-1 & 4.0 & $10^{6}$ & 0.01 & 2.07 & $\left(5.1_{-0.5}^{+0.8}\right) \times 10^{2}$ & $\left(6.9_{-0.7}^{+0.6}\right) \times 10^{3}$ & $40 \pm 4$ \\
\hline SextansA/diff-2 & 5.0 & $10^{6}$ & 0.00 & 0.14 & $\left(1.8_{-0.3}^{+0.3}\right) \times 10^{2}$ & $\left(8.6_{-0.7}^{+0.6}\right) \times 10^{3}$ & $30 \pm 8$ \\
\hline SextansA/diff-3 & 4.0 & $10^{6}$ & 0.01 & 7.20 & $\left(3.2_{-0.3}^{+0.6}\right) \times 10^{2}$ & $\left(6.6_{-0.7}^{+0.6}\right) \times 10^{3}$ & $37 \pm 6$ \\
\hline SextansA/diffuse & 2.5 & $10^{6}$ & 0.01 & 0.05 & $\left(3.1_{-0.4}^{+0.3}\right) \times 10^{3}$ & $\left(1.4_{-0.1}^{+0.1}\right) \times 10^{4}$ & $29 \pm 3$ \\
\hline ESO146-G14/disk & 1.5 & $10^{6}$ & 0.01 & 4.13 & $\left(5.9_{-0.5}^{+0.9}\right) \times 10^{5}$ & $\left(2.5_{-0.5}^{+0.1}\right) \times 10^{3}$ & $30 \pm 1$ \\
\hline ESO146-G14/sf-1 & 2.5 & $10^{6}$ & 0.01 & 3.45 & $\left(7.5_{-1.0}^{+2.1}\right) \times 10^{4}$ & $\left(1.6_{-0.5}^{+0.2}\right) \times 10^{3}$ & $44 \pm 12$ \\
\hline ESO146-G14/sf-2 & 4.0 & $10^{6}$ & 0.01 & 0.19 & $\left(6.2_{-0.8}^{+0.9}\right) \times 10^{4}$ & $\left(2.7_{-0.5}^{+0.2}\right) \times 10^{3}$ & $31 \pm 5$ \\
\hline ESO146-G14/sf-3 & 0.7 & $10^{6}$ & 0.01 & 3.65 & $\left(2.7_{-0.2}^{+0.2}\right) \times 10^{5}$ & $\left(5 ._{-4.7}^{+2.2}\right) \times 10^{2}$ & $28 \pm 4$ \\
\hline ESO146-G14/diffuse & 0.7 & $10^{6}$ & 0.12 & 1.77 & $\left(2.5_{-0.3}^{+0.3}\right) \times 10^{5}$ & $\left(4.4_{-0.5}^{+0.2}\right) \times 10^{3}$ & $25 \pm 7$ \\
\hline
\end{tabular}

Extended Data Table $4 \mid$ The fitting results: Key derived parameters from fitting the dust model to the m1 flux measurements of Extended Data Table 1 and 2. In addition to the flux errors reported in Extended Data Table 1, the uncertainties in the aboslute flux calibration are added before performing the fits, as described in the text. The last column is the dust temperature as given by modified black-body fitting.

\begin{tabular}{|c|c|c|c|c|c|}
\hline region & $\begin{array}{l}\log \Sigma_{\text {gas }}^{\mathrm{m} 1-M W} \\
\left(\log \mathrm{M}_{\odot} / \mathrm{pc}^{2}\right)\end{array}$ & $\begin{array}{l}\log \Sigma_{\text {gas }}^{m 2-M W} \\
\left(\log M_{\odot} / \mathrm{pc}^{2}\right)\end{array}$ & $\begin{array}{l}\log \Sigma_{\text {gas }}^{\text {ma-MW }} \\
\left(\log M_{\odot} / \mathrm{pc}^{2}\right)\end{array}$ & $\begin{array}{l}\log \Sigma_{\text {gas }}^{\mathrm{m} 1-S M C} \\
\left(\log M_{\odot} / \mathrm{pc}^{2}\right)\end{array}$ & $\begin{array}{l}\log \sum_{\mathrm{gas}}^{\mathrm{m} 1-\mathrm{LMC} 2} \\
\left(\log \mathrm{M}_{\odot} / \mathrm{pc}^{2}\right)\end{array}$ \\
\hline SextansA/sf-1 & $2.26_{-0.22}^{+0.23}$ & $2.10_{-0.22}^{+0.23}$ & $2.39_{-0.22}^{+0.24}$ & $2.24_{-0.22}^{+0.44}$ & $2.19_{-0.22}^{+0.57}$ \\
\hline SextansA/sf-2 & $2.57_{-0.21}^{+0.21}$ & $2.40_{-0.21}^{+0.22}$ & $2.71_{-0.22}^{+0.22}$ & $2.62_{-0.22}^{+0.22}$ & $2.65_{-0.21}^{+0.22}$ \\
\hline SextansA/sf-3 & $2.21_{-0.21}^{+0.23}$ & $2.05_{-0.21}^{+0.21}$ & $2.35_{-0.22}^{+0.22}$ & $2.25_{-0.21}^{+0.22}$ & $2.31_{-0.22}^{+0.22}$ \\
\hline SextansA/sf-4 & $2.46_{-0.21}^{+0.21}$ & $2.30_{-0.21}^{+0.21}$ & $2.59_{-0.22}^{+0.21}$ & $2.52_{-0.22}^{+0.23}$ & $2.55_{-0.22}^{+0.22}$ \\
\hline ESO146-G14/sf-1 & $1.21_{-0.22}^{+0.24}$ & $1.21_{-0.22}^{+0.23}$ & $1.25_{-0.22}^{+0.24}$ & $1.14_{-0.22}^{+0.23}$ & $1.15_{-0.24}^{+0.23}$ \\
\hline ESO146-G14/sf-2 & $1.12_{-0.22}^{+0.22}$ & $1.12_{-0.21}^{+0.23}$ & $1.16_{-0.21}^{+0.23}$ & $1.09_{-0.22}^{+0.22}$ & $1.16_{-0.24}^{+0.24}$ \\
\hline ESO146-G14/sf-3 & $1.77_{-0.21}^{+0.21}$ & $1.77_{-0.21}^{+0.22}$ & $1.80_{-0.21}^{+0.22}$ & $1.82_{-0.22}^{+0.22}$ & $1.80_{-0.24}^{+0.22}$ \\
\hline
\end{tabular}

Extended Data Table 5 | Gas mass surface densities by models of different dust types: Gas surface densities were derived from the dust masses that are based on IR SED fitting by dust models of Milky-Way (MW), Small Magellanic cloud (SMC) and Large Magellanic cloud (LMC) grains.

\begin{tabular}{lll}
\hline region & $\begin{array}{l}I_{\mathrm{CO}} \\
(\mathrm{K} \mathrm{km} / \mathrm{s})\end{array}$ & $\begin{array}{l}f_{\mathrm{H}_{2}}(S(1)-17.035 \mu \mathrm{m}) \\
\left.(\mathrm{W} \mathrm{m})^{-2}\right)\end{array}$ \\
\hline SextansA/sf-1 & 0.33 & $2.0 \mathrm{E}-17$ \\
SextansA/sf-2 & 0.67 & $1.5 \mathrm{E}-16$ \\
SextansA/sf-3 & 0.25 & $6.1 \mathrm{E}-16$ \\
SextansA/sf-4 & 0.56 & $1.1 \mathrm{E}-16$ \\
ESO146-G14/sf-1 & 0.02 & $4.2 \mathrm{E}-18$ \\
ESO146-G14/sf-2 & 0.01 & $1.1 \mathrm{E}-17$ \\
ESO146-G14/sf-3 & 0.10 & $5.4 \mathrm{E}-18$ \\
\hline
\end{tabular}

Extended Data Table 6 | Predicted $\mathrm{CO}$ and warm $\mathrm{H}_{2}$ line fluxes. 


\section{SextansA (360"x360")}
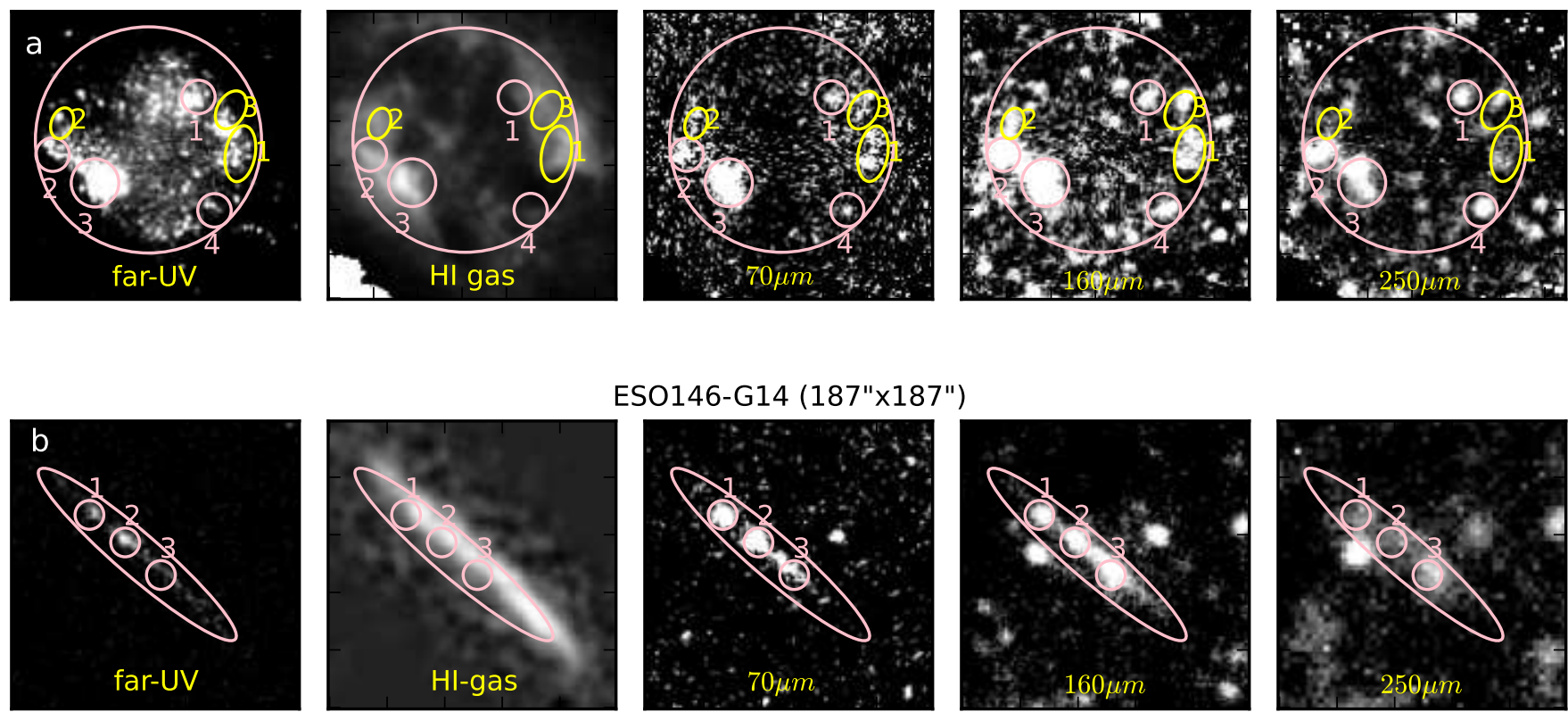

ESO146-G14 (187"x187")
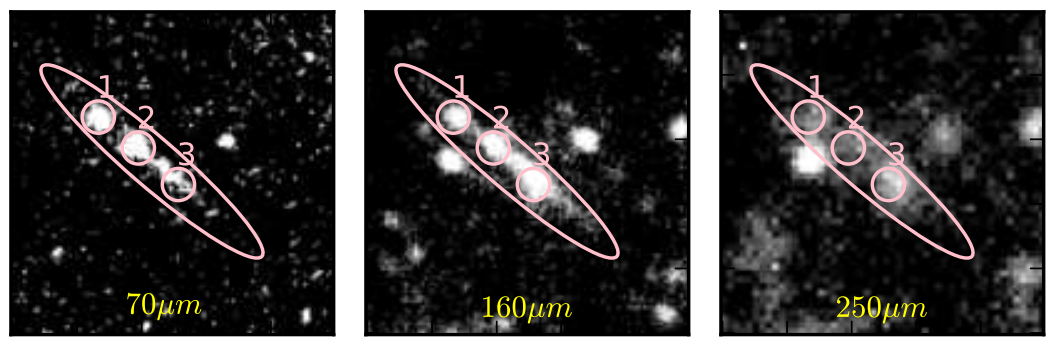

Extended Data Figure 1 | Mutli-wavelength images of two galaxies: a, Images of Sextans A in the far-UV, HI gas, $70 \mu \mathrm{m}, 160 \mu \mathrm{m}$ and $250 \mu \mathrm{m}$ dust emission, respectively. The large circle is the star-forming disk, small circles are star-forming clumps while ellipses are diffuse regions. b, Images of ESO 146-G14 in the far-UV, $\mathrm{HI}$ gas, $70 \mu \mathrm{m}, 160 \mu \mathrm{m}$ and $250 \mu \mathrm{m}$ dust emission, respectively. The large ellipse is the star-forming disk and small circles are individual star-forming clumps. 